\title{
Intensity interferometry with the MAGIC telescopes
}

\author{
Carlos Delgado, ${ }^{a, *}$ V.A. Acciari, ${ }^{b}$ E. Colombo, ${ }^{b}$ J. Cortina, ${ }^{a}$ C. Diaz, ${ }^{a}$ D. Fink, ${ }^{c}$ \\ M. Fiori, ${ }^{e}$ T. Hassan, ${ }^{a}$ I. Jiménez-Martínez, ${ }^{a}$ E. Lyard, ${ }^{d}$ S. Mangano, ${ }^{a}$ R. Mirzoyan, ${ }^{c}$ \\ G. Naleto, ${ }^{e}$ T. Njoh Ekoume, ${ }^{b}$ M. Polo, ${ }^{a}$ N. Produit, ${ }^{d}$ JJ. Rodriguez, ${ }^{a}$ T. Schweizer, ${ }^{c}$ \\ R. Walter, ${ }^{d}$ C. Wunderlich ${ }^{f}$ and L. Zampieri ${ }^{g}$ on behalf of the MAGIC Collaboration ${ }^{\dagger}$ \\ ${ }^{a}$ CIEMAT, \\ Madrid, Spain \\ ${ }^{b}$ IAC \& University of La Laguna, \\ La Laguna, Spain \\ ${ }^{c}$ Max-Planck-Institut for Physics, \\ Munich, Germany \\ ${ }^{d}$ University of Geneva, Department of Astronomy, \\ Geneva Switzerland \\ ${ }^{e}$ INFN-Padova \& Padova University, Department of Physics and Astronomy \\ Padova, Italy \\ ${ }^{f}$ INFN-Pisa \& Siena University, \\ Pisa, Italy \\ ${ }^{g}$ INAF - Astronomical Observatory of Padova, \\ Padova, Italy \\ E-mail: carlos.delgado@ciemat.es
}

Due to their large mirror size, fast response to single photons, sensitivity and telescope baselines in the order of $100 \mathrm{~m}$, Imaging Atmospheric Cherenkov Telescopes are ideally suited to perform intensity interferometry observations. In 2019 a test readout setup was installed in the two 17-m diameter MAGIC telescopes to allow performing interferometry measurements with them. The first on-sky measurements were able to detect correlated intensity fluctuations consistent with the stellar diameters of three different stars: Adhara ( $\epsilon \mathrm{CMa}$ ), Benetnasch ( $\eta \mathrm{UMa})$ and Mirzam ( $\beta$ CMa). After the upgrade of the setup in 2021, MAGIC is now equipped with a high-duty-cycle intensity interferometer, already in operation. A technical description of the interferometer and first performance results obtained by measuring several known stellar diameter are presented.

$37^{\text {th }}$ International Cosmic Ray Conference (ICRC 2021)

July 12 th - 23rd, 2021

Online - Berlin, Germany

\footnotetext{
${ }^{*}$ Presenter

${ }^{\dagger}$ a complete list of the MAGIC Collaboration authors can be found at the end of the proceedings
} 


\section{Introduction}

Intensity interferometry is an experimental technique, pioneered by Hanbury Brown and Richard Q. Twiss[1], that allows to infer properties of image of thermal sources in the optical band with milliarcsecond (mas) resolution. It exploits the time-correlation of the instantaneous intensity of light observed at two or more separated locations. Contrary with the more common amplitude interferometry, it is practically insensitive to optical imperfections of the telescope and atmospheric turbulence.

Inferring of properties of the image of the source is possible through the Van Cittert-Zernike theorem together with its relationship with the second-order correlation function. The Van CittertZernike and its generalizations state that for thermal sources and narrow band light, the mutual coherence of the electromagnetic field at two given points, $\gamma_{1,2}$, is proportional to the Fourier Transform of the intensity pattern of the source, evaluated at the distance between these two points measured in wavelengths of the field. On the other hand, the second-order intensity correlation function $g_{1,2}$ [2] on these two given points for non-polarized light relates to the mutual coherence accordingly to the following equation

$$
g_{1,2}=\frac{\left\langle I_{1}(t) I_{2}(t+\tau)\right\rangle_{t}}{\left\langle I_{1}(t)\right\rangle_{t}\left\langle I_{2}(t)\right\rangle_{t}}=1+\frac{\Delta f}{\Delta v}\left|\gamma_{1,2}(\tau)\right|^{2}
$$

where $I_{i}(t)$ is the light intensity at point $i$ at time $t,\langle x\rangle_{t}$ denotes time average of $x, \Delta f$ is the electronics bandwidth, $\Delta v$ is the optical bandwidth, and $\tau$ is the time shift between the two signal of the evaluation of the coherence. This relationship enables to measure the modulus of the coherence function by means of counting the coincidence in time of detection of photons from a source at two separated points. In particular for a source described by a disc of uniform intensity up to an angular diameter $\theta,\left|\gamma_{1,2}\right|$ has a simple expression:

$$
\left|\gamma_{1,2}\right|=2 \frac{B_{1}\left(\pi \cdot d_{1,2} \cdot \theta / \lambda\right)}{\pi \cdot d_{1,2} \cdot \theta / \lambda}
$$

where $B_{1}$ is the Bessel function of first order, $d_{1,2}$ is the distance between the optical axes from these points to the source, called baseline, and $\lambda$ is the light wavelength. From this equation, it is clear that it is possible to infer the angular diameter of the source by measuring

$$
\frac{g_{1,2}-1}{g_{0}-1}=\frac{\left|\gamma_{1,2}\right|^{2}}{\left|\gamma_{0}\right|^{2}}
$$

where $\gamma_{0}$ and $g_{0}$ denote the coherence and the second-order intensity correlation at zero baseline respectively.

When detecting a source using this experimental technique with two telescopes at point 1 and 2 using filtered light around a given wavelength $\lambda$, the signal to noise ratio of the measurement is given by [1]

$$
S / N=\Phi \cdot A \cdot O \cdot Q \cdot\left|\gamma_{1,2}\right|^{2} \cdot \sqrt{B \cdot T / 2} \cdot \sigma / F
$$

where $\Phi$ is time-averaged differential source flux flux at wavelength $\lambda, A$ is the geometric mean of the mirror areas of the telescopes, $O$ and $Q$ are the optical efficiency (including the atmospheric transmittance) and quantum efficiency of the system at $\lambda$ respectively, $B$ is the cross-correlation 
electrical bandwidth, $T$ the observation time, $\sigma$ is the normalized spectral distribution of the light as defined in formula 5.6 of [1], and $F$ is the photodetectors excess noise factor.

From eq. 4 it is clear that intensity interferometry can be realized with large mirror sized telescopes equipped with fast and sensitive electronics, making Imaging Atmospheric Cherenkov Telescopes (IACTs), in particular the 17 meters diameter MAGIC ones, well suited platforms for the implementation of intensity interferometers and the measurement of the diameter of stars[3]. Given the typical distances between IACTs and the typical wavelength it can detect, eq. 2 predicts it could reach resolutions of the order of 1 mas. This results in relatively bright and hot, single or binary $\mathrm{O}, \mathrm{B}$, and WR-type stars to be natural targets to observe with an IACTs based intensity interferometry to study their dynamics[4]. In this paper we describe an intensity interferometer implementation in the MAGIC telescopes, as well as its acquisition system. In addition we briefly sketch the observed performance derived from real observation.

\section{MAGIC telescopes}

MAGIC is a system of two IACTs located at the Roque de los Muchachos Observatory (Observatorio del Roque de los Muchachos, ORM) at the island of La Palma in Spain[5]. Equipped with 17-m diameter mirror dishes and fast PMT cameras, the telescopes record images of extensive air showers in stereoscopic mode, enabling the observation of VHE $\gamma$-ray sources at energies $\gtrsim 50$ $\mathrm{GeV}$. Each telescope is equipped with a 1039-pixel PMT camera at the primary focus. The PMTs are $25.4 \mathrm{~mm}$ diameter, optically coupled with a hexagonal shaped Winston cone, such that each pixel covers a $0.1^{\circ}$ of the FOV. The distance between PMT centers is $30 \mathrm{~mm}$. The PMTs are Hamamatsu, type R10408, with a hemispherical photocathode and 6 dynodes. The PMT bias voltages for the cathode and dynodes are generated by a Cockroft-Walton DC-DC converter, which can provide up to $1250 \mathrm{~V}$ peak voltage. The electrical signals are amplified (AC coupled, $\approx 25 \mathrm{~dB}$ amplification) and then transmitted via independent optical fibers by means of vertical cavity surface emitting lasers (VCSELs). The average pulse width signal is measured to be $2.5 \mathrm{~ns}$ (FWHM).

The telescopes reflectors follows a parabolic shape to minimize the time spread at the focal plane, with a F/D ratio of 1 . The reflector is formed by $\approx 2501 \mathrm{~m}^{2}$ spherical mirror tiles. Two actuators behind each tile can be used to shift its orientation and correct the reflector for deformations in its parabolic shape as the telescope changes zenith. This so-called Active Mirror Control (AMC) adjusts the mirror in a few seconds, and runs typically every 20 minutes during standard observations. Approximately $70 \%$ of the light of a point source is focused on a pixel. It must be noted that individual mirror tiles are slightly staggered thus introducing an extra time spread in the order of 200 ps.

\section{Implementation of an intensity interferometer in MAGIC}

The implementation of the intensity interferometer builds up on top of the actual MAGIC hardware. It improves the DAQ efficiency and duty cycle of the proof of concept of reference [6], and provides near realtime feedback of the observed sources.

In order to avoid damaging the MAGIC PMTs when pointing to bright stars or during observations with bright Moon, the interferometer places a filter $15 \mathrm{~mm}$ in front of the Winston cone 

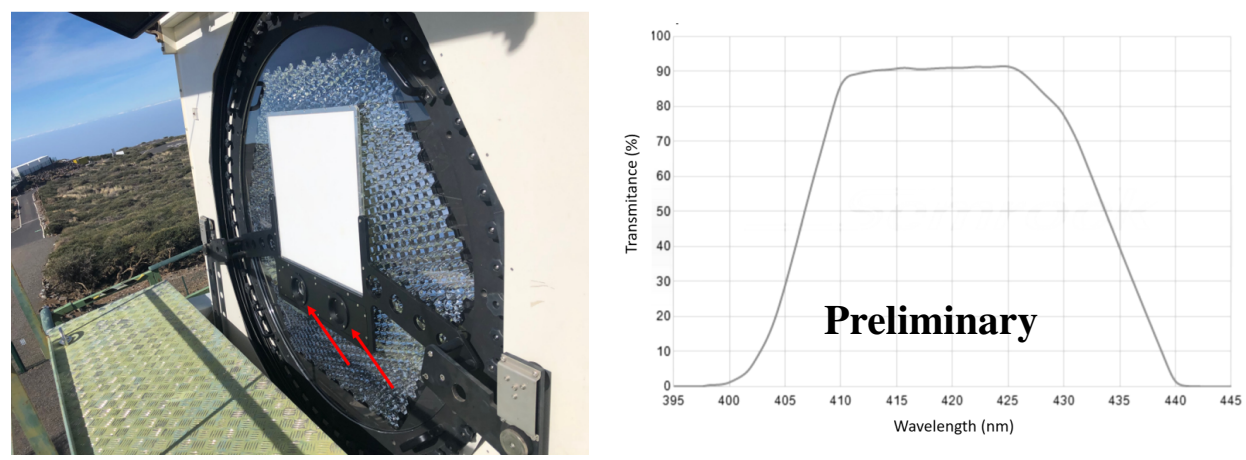

Figure 1: (left) Picture of the target holder deployed in one of the MAGIC telescopes camera. The placement of the filters for two pixels are marked with red arrows.(right) Effective transmittance of the filters as a function of the light wavelength.

for each one of the pixels used for interferometry observations. These filters are held on place by a custom permanent support structure mounted on the automatic target holder of MAGIC used for calibration of its focusing, as shown in the left panel of fig. 1. The automatic deployment of this holder places the necessary filters without requiring any manual intervention on the camera. Two Semrock filters per camera are installed, enabling the use of two different pixels on each camera for interferometrys. Their transmission is larger than $90 \%$ for a wavelength between 410 and $425 \mathrm{~nm}$ for perpendicular incident light. The dependence on the incidence angle of the filter transmission convoluted with the light angular distribution for MAGIC cameras results in the effective transmission shown in the right panel of fig. 1, which translates into a reduction of the sensitivity of the interferometer by about $15 \%$ compared with perpendicular incidence.

MAGIC optical link used to carry the signal of the two pixels out of the four available (two per camera) selected to observe with the interferometer to a digitizer board installed in an interferometry dedicated server. Default observations use one pixel per camera, resulting in baselines of several tens to 86 meters (termed long baseline observations). However by making use of MAGIC AMC to focus arbitrary sections of the telescopes mirror to different pixels it is possible to perform observations with baselines ranging from zero to $\approx 15$ meters with two pixels in a single telescope (termed short baselines observations respectively)

The signal of the two pixels reaching the interferometer server, a dual processor Xeon machine with 20 cores, 192 MBytes of RAM, 32 TBytes of SSD and 80 TBytes of HD, is digitised by a two channel spectrum M4i.4450-x8 digitizer card, which samples at 500MS/s in continuous mode simultaneously for both channels, with a resolution of 14bits. The resulting data streams are sent to data consumers via a PCIExpress bus, resulting in a writing speed of about $2 \mathrm{~GB} / \mathrm{s}$.

The acquisition software implements two modes. The debugging mode writes the streams in the SSD disk, allowing for subsequent offline analysis of waveform for each channel as well as computation of the correlation for arbitrary stream lengths. However, this mode has two clear drawbacks, its large $2 \mathrm{~GB} / \mathrm{s}$ data throughput and the latency in obtaining a feedback on the correlation measurement. On the other hand, the data-reduction mode streams the data to a NVIDIA Tesla V100 GPU card where a realtime analysis allows to compute the correlation of the two channels. The data-reduction mode is implemented by splitting the continuous stream in contiguous frames of $2^{18}$ samples/channel. For each frame, the Pearson's correlation coefficient between the channels as 


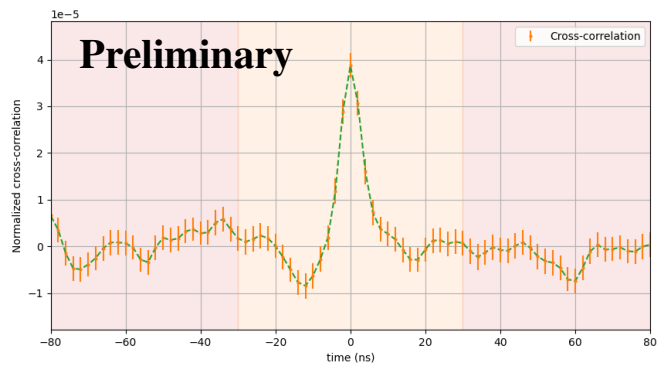

Figure 2: Measured 10 minutes average correlation histogram for a star with a brightness of $\approx 1.3 \mathrm{mag}$ in $\mathrm{B}$, as a function of the time shift between the two signals. A shift of zero corresponds to the expected position for the correlation. The error bars represent standard deviation of the average. The yellow region represents the region of interest used to look for the correlation signal. The dashed line is just to guide the eye. This correlation histogram is obtained in real-time, as the data is being recorded in disk.

a function of a time shift $\tau$ between the channels, $\rho(\tau)$, is computed using Fourier Transforms. The values for each frame are stored on disk for time shifts between -2048 and $2048 \mathrm{~ns}$, in bins of $2 \mathrm{~ns}$. This pipeline results in a throughput reduction of a factor 64, providing the correlation in real time, in a region of time shifts large enough to study sources of electronic noise, and to accommodate possible uncertainties in the expected delay between the two signals being correlated.

\section{Analysis and observations}

The Pearson's correlation coefficients, provided by the data-reduction acquisition mode, are subsequently analyzed in real-time to determine the presence of a correlation. As an example, figure 2 shows the obtained average correlation histogram for 10 minutes of observation of a star with a brightness of $\approx 1.3 \mathrm{mag}$ in $\mathrm{B}$, showing the correlation at the expected time delay between the two interferometer channels.

The steps to get from the Pearson's correlation histogram to the second-order intensity correlation function are described in [6]: in the approximation of fluctuations on the digitized channels being dominated by Poissonian fluctuations of the source ${ }^{1}$, and that the signals has no contribution from other sources beyond the one observed, it can be shown that

$$
\left|\gamma_{1,2}\right|^{2}=K \frac{\rho_{1,2}}{\sqrt{D C_{1} D C_{2}}}
$$

where $K$ is a constant, and $D C_{1}$ and $D C_{2}$ are the anode current of the two digitized photodetectors. $D C_{1}$ and $D C_{2}$ are measured in MAGIC roughly every second and hardly change in time scales of a few seconds, but depend strongly on the star and observation conditions on a longer time scale. In presence of the strong diffuse background of the Moonlight, eq. 5 must be corrected by subtracting the expected anode current produced by the Moon in each individual photodetector. Simultaneous off-source DC measurements are taken using the second available optical filter socket in each telescope to estimate and substract the DC associated to the Moon. Finally the correlation histogram is time shifted to account for difference of the time arrival to the two photomultipliers of a signal originating in the source due to the pointing direction.

\footnotetext{
1In the context of intensity interferometry, this is valid for Pearson's correlation coefficients much smaller than one.
} 

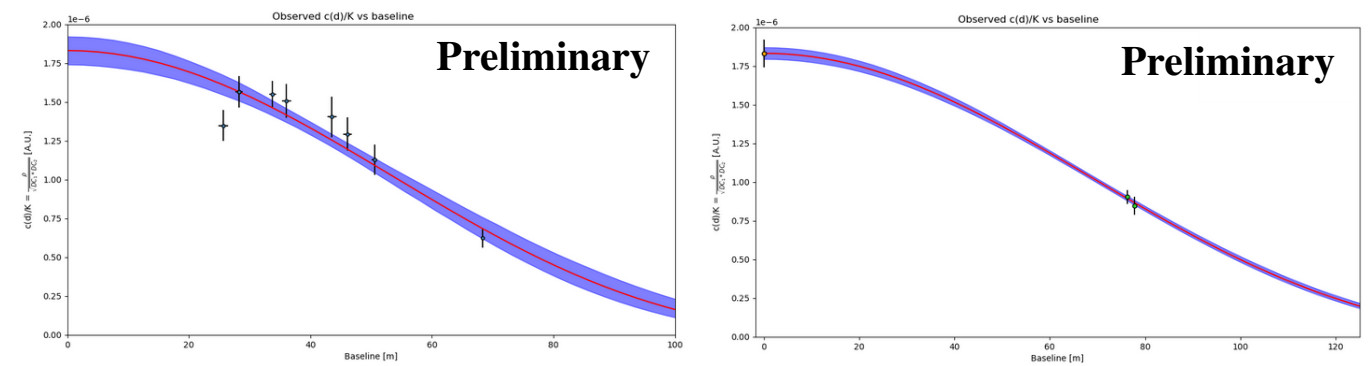

Figure 3: (left) Fit of eq. 5 to expected shape given by eq. 2 for the correlation of a calibrator star. The red curve is the most probable value of the fit, whereas the filled area represents the variance of the fitted function. The zero baseline correlation, or equivalently $K$ in eq. 5 , is the value of the fit evaluated at zero. (right) Fit of the measured correlation as a function of the baseline to another star, where the zero baseline correlation has been constrained by the measurement in the left panel.

The amplitude of the correlation signal is extracted by fitting a Gaussian function to the resulting corrected correlation histogram, built with continuous observations of minute scales, such that the baseline changes due to the source tracking of the order of few meters, and the $\mathrm{S} / \mathrm{N}$ is large to observe the correlation. The dependence of this signal with the baseline distance is fitted to eqs. 2 and 3 with $\theta$, the source diameter, as free parameters. As discussed in the introduction, the other parameter of this fit, $K$ in eq. 5, only depends on the instrumental setup. It can be calibrated by observing calibration stars and performing a fit leaving as free parameters both $K$ and $\theta$. The calibrator stars are bright ones which can be observed in a large range of baselines and have a stellar diameter between 0.1 and 1.0 mas. The resulting value of $K$ obtained in this manner is used to constrain the fit for other stars. The left panel of fig. 3 illustrates the calibration measurement results and the fit for one calibrator star. The right panel of the same figure shows the use of the obtained zero baseline correlation value to constrain the fit of other star, that enables the measurement of the stellar diameter of the latter with improved errors.

The observations performed with the implemented interferometer observe long or short baselines, depending on the pixel signals injected in the interferometry server.

For the long baseline observations, we inject the signal of a single pixel of each MAGIC telescope into the interferometer acquisition server. This allows to cover baselines between few tens to $\approx 86$ meters. In order to study the performance of this particular setup with the analysis described above, we have measured the stellar diameter of 11 stars with known diameters between 0.2 and 0.8 mas, and brightness between 1.2 and 3.8 mag in B. All observations took place with high Moon diffuse background in two campaigns. The total observation time for each star was 30 minutes. The zero baseline correlation was constrained by using the brightest star of the set as calibrator.

Figure 4 shows the resulting deviation of the measured stellar diameters with respect to the reference value in this set, showing a good general agreement even if the analysis is not fully optimized.

Short baselines observations are still in the proof of concept phase. They are achieved by injecting the signal of two pixels of the same MAGIC telescope in the interferometer server, and focusing different sections of its mirror into these pixels, thanks to the features of the AMC system. These measurements have two goals, a) performing a direct measurement of the zero baseline correlation so that there is no need to rely on other fits (as described in the previous section); and 


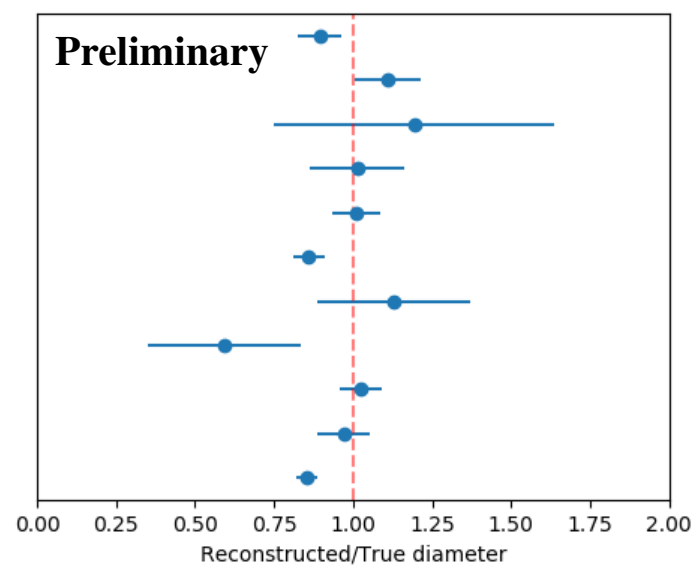

Figure 4: Deviation of the measured stellar diameter with respect the known ones. The errors account for the uncertainty of our measurement. The vertical red line is just to guide the eye.

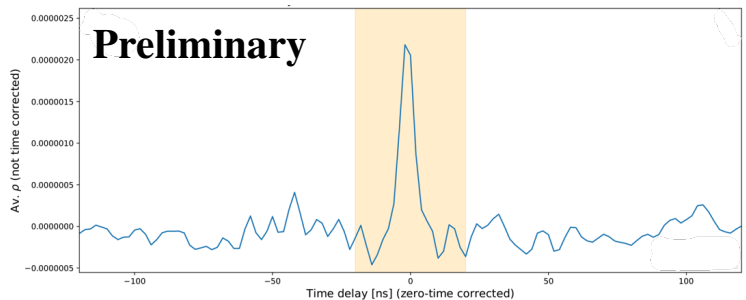

Figure 5: Illustration of the measurement of the zero baseline correlation using the procedure described in the text. The shaded region shows the region of interest to extract the zero baseline correlation.

b) expanding the maximum angular size of stars MAGIC is able to study by narrowing down the shortest baselines accessible to our telescopes (down to $\approx 20 \mathrm{~m}$ in long-baseline mode).

To measure the zero baseline correlation, the individual facets of the mirror of the telescope are split in two alternating sets in a chessboard fashion, and each set is focused in a single pixel. The corresponding baseline between each set is effectively zero, so the measured correlation of any bright enough star provides this parameter. Figure 5 shows the obtained correlation histogram in a test run in this mode. The zero baseline correlation is extracted by performing the Gaussian fit explained in the previous section, which results in a value compatible within errors with the one obtained with a calibrator star with the long baseline observation.

To measure the diameter of large and bright stars, a small set of the mirror facets are split in two small size mirrors of few square meters away, focused to two pixels, as illustrated in left panel of fig. 6. The resulting fit to the correlation against the baseline of a test run observing $\alpha \mathrm{CMa}$ is shown in the right panel of the same figure, which results in a stellar diameter of $6.2 \pm 0.6$ mas, compatible with the value of $6.05 \pm 0.05$ mas[7].

\section{Conclusions and outlook}

We have presented the MAGIC intensity interferometer hardware, data acquisition system, observation modes and a brief description of its performance in real conditions. We have proved that measurements can be routinely performed on IACTs with an automatic deployment of optical 

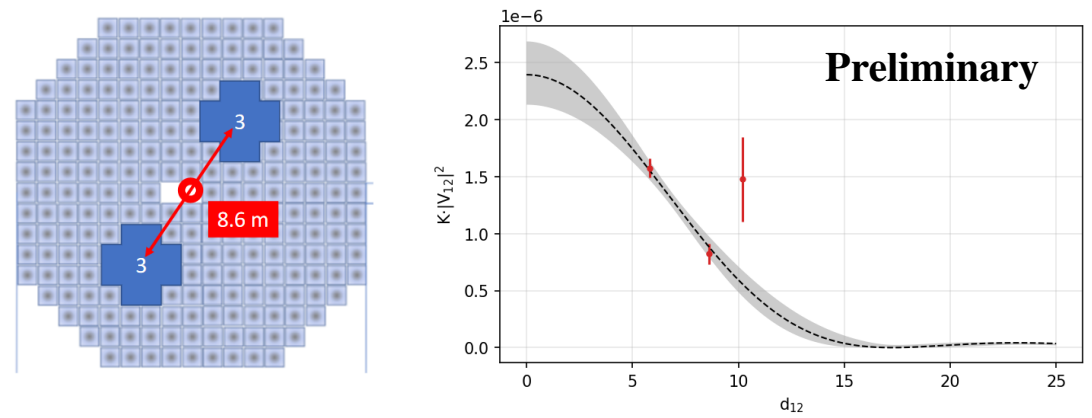

Figure 6: (left) sketch showing the two sub-mirrors that are used for a correlation measurement with a baseline of $8.6 \mathrm{~m}$. The $1 \mathrm{~m}^{2}$ individual facets in the telescope mirror are colored in light blue. The two groups of facets colored in deep blue and marked with a 3 are focused on two different pixels. (right) Fit to the correlation as a function of the baseline for $\alpha \mathrm{CMa}$.

filter. We have proved as well that correlations of different pixels within the same camera are possible in IACTs with AMC systems, opening new future possibilities.

\section{Acknowledgements}

We would like to acknowledge F. Vakili and P. M. Gori for the fruitful discussion about the potential of MAGIC to implement and exploit a short baseline interferometer. We acknowledge the support from the agencies and organizations listed at https://magic.mpp.mpg.de/acknowledgments_ICRC2021.

\section{References}

[1] Hanbury Brown, R., The Intensity Interferometer. Its applications to astronomy, Taylor \& Francis, London(1974)

[2] R. J. Glauber, Quantum theory of optical coherence, Phys. Rev. 130, 2529 (1963)

[3] Abeysekara, A.U., Benbow, W., Brill, A. et al.Demonstration of stellar intensity interferometry with the four VERITAS telescopes. Nat Astron 4, 1164-1169 (2020)

[4] Dravins, D. et al. Optical Intensity Interferometry with the Cherenkov Telescope Array. Astropart. Phys. 43 (2012)

[5] Aleksic J., et al., The major upgrade of the MAGIC telescopes, Part II: A performance study using observations of the Crab Nebula, Astropart. Phys. 72 (2016)

[6] Acciari, V.A. et al. Optical intensity interferometry observations using the MAGIC Imaging Atmospheric Cherenkov Telescopes, MNRAS, 491, 2, (2020)

[7] Davis, J., et al., The Angular Diameter and Fundamental Parameters of Sirius A, Publications of the Astronomical Society of Australia, 28 (2011) 


\section{The MAGIC Collaboration}

V. A. Acciari ${ }^{1}$, S. Ansoldi ${ }^{2,41}$, L. A. Antonelli ${ }^{3}$, A. Arbet Engels $^{4}$, M. Artero ${ }^{5}$, K. Asano ${ }^{6}$, D. Baack ${ }^{7}$, A. Babić ${ }^{8}$, A. Baquero ${ }^{9}$, U. Barres de Almeida ${ }^{10}$, J. A. Barrio ${ }^{9}$, I. Batković ${ }^{11}$, J. Becerra González ${ }^{1}$, W. Bednarek ${ }^{12}$, L. Bellizzi ${ }^{13}$, E. Bernardini ${ }^{14}$, M. Bernardos ${ }^{11}$, A. Berti ${ }^{15}$, J. Besenrieder ${ }^{15}$, W. Bhattacharyya ${ }^{14}$, C. Bigongiari ${ }^{3}$, A. Biland ${ }^{4}$, O. Blanch ${ }^{5}$, H. Bökenkamp ${ }^{7}$, G. Bonnoli ${ }^{16}$, Ž. Bošnjak $^{8}$, G. Busetto ${ }^{11}$, R. Carosi ${ }^{17}$, G. Ceribella ${ }^{15}$, M. Cerruti ${ }^{18}$, Y. Chai ${ }^{15}$, A. Chilingarian ${ }^{19}$, S. Cikota ${ }^{8}$, S. M. Colak ${ }^{5}$, E. Colombo ${ }^{1}$, J. L. Contreras ${ }^{9}$, J. Cortina ${ }^{20}$, S. Covino ${ }^{3}$, G. D’Amico ${ }^{15,42}$, V. D’Elia ${ }^{3}$, P. Da Vela ${ }^{17,43}$, F. Dazzi ${ }^{3}$, A. De Angelis ${ }^{11}$, B. De Lotto ${ }^{2}$, M. Delfino ${ }^{5,44}$, J. Delgado ${ }^{5,44}$, C. Delgado Mendez ${ }^{20}$, D. Depaoli ${ }^{21}$, F. Di Pierro ${ }^{21}$, L. Di Venere ${ }^{22}$, E. Do Souto Espiñeira ${ }^{5}$, D. Dominis Prester $^{23}$, A. Donini ${ }^{2}$, D. Dorner ${ }^{24}$, M. Doro ${ }^{11}$, D. Elsaesser ${ }^{7}$, V. Fallah Ramazani ${ }^{25,45}$, A. Fattorini ${ }^{7}$, M. V. Fonseca ${ }^{9}$, L. Font ${ }^{26}$, C. Fruck ${ }^{15}$, S. Fukami ${ }^{6}$, Y. Fukazawa ${ }^{27}$, R. J. García López ${ }^{1}$, M. Garczarczyk ${ }^{14}$, S. Gasparyan ${ }^{28}$, M. Gaug ${ }^{26}$, N. Giglietto ${ }^{22}$, F. Giordano ${ }^{22}$, P. Gliwny ${ }^{12}$, N. Godinović ${ }^{29}$, J. G. Green ${ }^{3}$, D. Green ${ }^{15}$, D. Hadasch ${ }^{6}$, A. Hahn ${ }^{15}$, L. Heckmann ${ }^{15}$, J. Herrera ${ }^{1}$, J. Hoang ${ }^{9,46}$, D. Hrupec ${ }^{30}$, M. Hütten ${ }^{15}$, T. Inada ${ }^{6}$, K. Ishio ${ }^{12}$, Y. Iwamura ${ }^{6}$, I. Jiménez Martínez ${ }^{20}$, J. Jormanainen ${ }^{25}$, L. Jouvin ${ }^{5}$, M. Karjalainen ${ }^{1}$, D. Kerszberg ${ }^{5}$, Y. Kobayashi ${ }^{6}$, H. Kubo ${ }^{31}$, J. Kushida ${ }^{32}$, A. Lamastra ${ }^{3}$, D. Lelas ${ }^{29}$, F. Leone ${ }^{3}$, E. Lindfors ${ }^{25}$, L. Linhoff ${ }^{7}$, S. Lombardi ${ }^{3}$, F. Longo ${ }^{2,47}$, R. López-Coto ${ }^{11}$, M. López-Moya ${ }^{9}$, A. López-Oramas ${ }^{1}$, S. Loporchio ${ }^{22}$, B. Machado de Oliveira Fraga $^{10}$, C. Maggio ${ }^{26}$, P. Majumdar ${ }^{33}$, M. Makariev ${ }^{34}$, M. Mallamaci ${ }^{11}$, G. Maneva ${ }^{34}$, M. Manganaro ${ }^{23}$, K. Mannheim ${ }^{24}$, L. Maraschi ${ }^{3}$, M. Mariotti ${ }^{11}$, M. Martínez ${ }^{5}$, D. Mazin ${ }^{6,15}$, S. Menchiari ${ }^{13}$, S. Mender ${ }^{7}$, S. Mićanović ${ }^{23}$, D. Miceli ${ }^{2,49}$, T. Miener $^{9}$, J. M. Miranda ${ }^{13}$, R. Mirzoyan ${ }^{15}$, E. Molina ${ }^{18}$, A. Moralejo ${ }^{5}$, D. Morcuende ${ }^{9}$, V. Moreno ${ }^{26}$, E. Moretti ${ }^{5}$, T. Nakamori ${ }^{35}$, L. Nava ${ }^{3}$, V. Neustroev ${ }^{36}$, C. Nigro $^{5}$, K. Nilsson ${ }^{25}$, K. Nishijima ${ }^{32}$, K. Noda $^{6}$, S. Nozaki ${ }^{31}$, Y. Ohtani' ${ }^{6}$, T. Oka ${ }^{31}$, J. Otero-Santos ${ }^{1}$, S. Paiano ${ }^{3}$, M. Palatiello ${ }^{2}$, D. Paneque ${ }^{15}$, R. Paoletti ${ }^{13}$, J. M. Paredes ${ }^{18}$, L. Pavletić ${ }^{23}$, P. Peñil ${ }^{9}$, M. Persic ${ }^{2,50}$, M. Pihet ${ }^{15}$, P. G. Prada Moroni ${ }^{17}$, E. Prandini ${ }^{11}$, C. Priyadarshi ${ }^{5}$, I. Puljak ${ }^{29}$, W. Rhode ${ }^{7}$, M. Ribó ${ }^{18}$, J. Rico ${ }^{5}$, C. Righi ${ }^{3}$, A. Rugliancich ${ }^{17}$, N. Sahakyan ${ }^{28}$, T. Saito ${ }^{6}$, S. Sakurai ${ }^{6}$, K. Satalecka ${ }^{14}$, F. G. Saturni ${ }^{3}$, B. Schleicher ${ }^{24}$, K. Schmidt ${ }^{7}$, T. Schweizer ${ }^{15}$, J. Sitarek ${ }^{12}$, I. Šnidaric ${ }^{37}$, D. Sobczynska ${ }^{12}$, A. Spolon ${ }^{11}$, A. Stamerra ${ }^{3}$, J. Strišković ${ }^{30}$, D. Strom ${ }^{15}$, M. Strzys ${ }^{6}$, Y. Suda ${ }^{27}$, T. Surić ${ }^{37}$, M. Takahashi ${ }^{6}$, R. Takeishi ${ }^{6}$, F. Tavecchio ${ }^{3}$, P. Temnikov ${ }^{34}$, T. Terzić ${ }^{23}$, M. Teshima ${ }^{15,6}$, L. Tosti ${ }^{38}$, S. Truzzi ${ }^{13}$, A. Tutone ${ }^{3}$, S. Ubach ${ }^{26}$, J. van Scherpenberg ${ }^{15}$, G. Vanzo ${ }^{1}$, M. Vazquez Acosta ${ }^{1}$, S. Ventura ${ }^{13}$, V. Verguilov ${ }^{34}$, C. F. Vigorito ${ }^{21}$, V. Vitale ${ }^{39}$, I. Vovk $^{6}$, M. Will ${ }^{15}$, C. Wunderlich ${ }^{13}$, T. Yamamoto $^{40}$, and D. Zarić 29

${ }^{1}$ Instituto de Astrofísica de Canarias and Dpto. de Astrofísica, Universidad de La Laguna, E-38200, La Laguna, Tenerife, Spain ${ }^{2}$ Università di Udine and INFN Trieste, I-33100 Udine, Italy ${ }^{3}$ National Institute for Astrophysics (INAF), I-00136 Rome, Italy ${ }^{4}$ ETH Zürich, CH-8093 Zürich, Switzerland ${ }^{5}$ Institut de Física d'Altes Energies (IFAE), The Barcelona Institute of Science and Technology (BIST), E-08193 Bellaterra (Barcelona), Spain ${ }^{6}$ Japanese MAGIC Group: Institute for Cosmic Ray Research (ICRR), The University of Tokyo, Kashiwa, 277-8582 Chiba, Japan ${ }^{7}$ Technische Universität Dortmund, D-44221 Dortmund, Germany ${ }^{8}$ Croatian MAGIC Group: University of Zagreb, Faculty of Electrical Engineering and Computing (FER), 10000 Zagreb, Croatia ${ }^{9}$ IPARCOS Institute and EMFTEL Department, Universidad Complutense de Madrid, E-28040 Madrid, Spain ${ }^{10}$ Centro Brasileiro de Pesquisas Físicas (CBPF), 22290-180 URCA, Rio de Janeiro (RJ), Brazil ${ }^{11}$ Università di Padova and INFN, I-35131 Padova, Italy ${ }^{12}$ University of Lodz, Faculty of Physics and Applied Informatics, Department of Astrophysics, 90-236 Lodz, Poland ${ }^{13}$ Università di Siena and INFN Pisa, I-53100 Siena, Italy ${ }^{14}$ Deutsches Elektronen-Synchrotron (DESY), D-15738 Zeuthen, Germany ${ }^{15}$ Max-Planck-Institut für Physik, D-80805 München, Germany ${ }^{16}$ Instituto de Astrofísica de Andalucía-CSIC, Glorieta de la Astronomía s/n, 18008, Granada, Spain ${ }^{17}$ Università di Pisa and INFN Pisa, I-56126 Pisa, Italy ${ }^{18}$ Universitat de Barcelona, ICCUB, IEEC-UB, E-08028 Barcelona, Spain ${ }^{19}$ Armenian MAGIC Group: A. Alikhanyan National Science Laboratory, 0036 Yerevan, Armenia ${ }^{20}$ Centro de Investigaciones Energéticas, Medioambientales y Tecnológicas, E-28040 Madrid, Spain ${ }^{21}$ INFN MAGIC Group: INFN Sezione di Torino and Università degli Studi di Torino, I-10125 Torino, Italy 22 INFN MAGIC Group: INFN Sezione di Bari and Dipartimento Interateneo di Fisica dell'Università e del Politecnico di Bari, I-70125 Bari, Italy ${ }^{23}$ Croatian MAGIC Group: University of Rijeka, Department of Physics, 51000 Rijeka, Croatia ${ }^{24}$ Universität Würzburg, D-97074 Würzburg, Germany ${ }^{25}$ Finnish MAGIC Group: Finnish Centre for Astronomy with ESO, University of Turku, FI-20014 Turku, Finland ${ }^{26}$ Departament de Física, and CERES-IEEC, Universitat Autònoma de Barcelona, E-08193 Bellaterra, Spain ${ }^{27}$ Japanese MAGIC Group: Physics Program, Graduate School of Advanced Science and Engineering, Hiroshima University, 739-8526 Hiroshima, Japan ${ }^{28}$ Armenian MAGIC Group: ICRANet-Armenia at NAS RA, 0019 Yerevan, Armenia ${ }^{29}$ Croatian MAGIC Group: University of Split, Faculty of Electrical Engineering, Mechanical Engineering and Naval Architecture (FESB), 21000 Split, Croatia ${ }^{30}$ Croatian MAGIC Group: Josip Juraj Strossmayer University of Osijek, Department of Physics, 31000 Osijek, Croatia ${ }^{31}$ Japanese MAGIC Group: Department of Physics, Kyoto University, 606-8502 Kyoto, Japan ${ }^{32}$ Japanese MAGIC Group: Department of Physics, Tokai University, Hiratsuka, 259-1292 Kanagawa, Japan ${ }^{33}$ Saha Institute of Nuclear Physics, HBNI, 1/AF Bidhannagar, Salt Lake, Sector-1, Kolkata 700064, India ${ }^{34}$ Inst. for Nucl. Research and Nucl. Energy, Bulgarian Academy of Sciences, BG-1784 Sofia, Bulgaria ${ }^{35}$ Japanese MAGIC Group: Department of Physics, Yamagata University, Yamagata 990-8560, Japan ${ }^{36}$ Finnish MAGIC Group: Astronomy Research Unit, University of Oulu, FI-90014 Oulu, Finland ${ }^{37}$ Croatian MAGIC Group: Ruđer Bošković Institute, 10000 Zagreb, Croatia ${ }^{38}$ INFN MAGIC Group: INFN Sezione di Perugia, I-06123 Perugia, Italy ${ }^{39}$ INFN MAGIC Group: INFN Roma Tor Vergata, I-00133 Roma, Italy ${ }^{40}$ Japanese MAGIC Group: Department of Physics, Konan University, Kobe, Hyogo 6588501, Japan ${ }^{41}$ also at International Center for Relativistic Astrophysics (ICRA), Rome, Italy ${ }^{42}$ now at Department for Physics and Technology, University of Bergen, NO-5020, Norway ${ }^{43}$ now at University of Innsbruck ${ }^{44}$ also at Port d'Informació Científica (PIC), E-08193 Bellaterra (Barcelona), Spain ${ }^{45}$ now at Ruhr-Universität Bochum, Fakultät für Physik und Astronomie, Astronomisches Institut (AIRUB), 44801 Bochum, Germany ${ }^{46}$ now at Department of Astronomy, University of California Berkeley, Berkeley CA $94720{ }^{47}$ also at Dipartimento di Fisica, Università di Trieste, I-34127 Trieste, Italy 49 now at Laboratoire d'Annecy de Physique des Particules (LAPP), CNRS-IN2P3, 74941 Annecy Cedex, France ${ }^{50}$ also at INAF Trieste and Dept. of Physics and Astronomy, University of 
Bologna, Bologna, Italy 